DELTA U N I VER S I T Y
SCIENTIFIC JOURNAL
Journal home page: www.deltauniv.edu.eg/dusj

\title{
التنبؤ بالأزمات المالية فى المصارف العراقية بإستخدام الثبكات العصبية
}

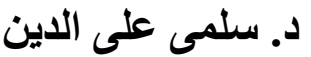

مدرس إدارة الأعمال

كلية الإدارة - جامعة الألتا للعلوم و التكنولوجيا

\author{
د. مصطفى يوسف الإسف

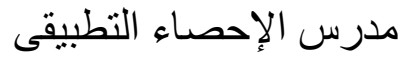 \\ كلية الإدارة - جامعة الالتا للعلوم و التكنولوجيا النطيا
}

\begin{abstract}
ملخص البحث:
يهدف البحث التتبؤ بالأزمات المالية فى المصارف العراقية باستخدام الثبكات العصبية، حيث تم التطبيق بإستخدام عينة من المصارف العراقية خلال الفترة

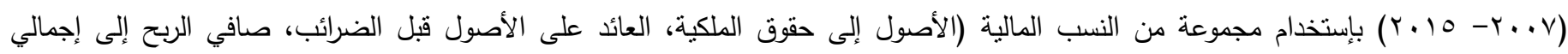

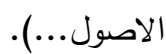

وقد توصل البحث الى أهمية استخدام الثبكات العصبية فى التتبؤ بالأزمات المالية حيث بلغت المساحة تحت منحنى Receiver Operator (ROC) Characteristic الكلمات الاليلية: الثبكات العصبية، الأزمات المالية، التتبؤ، منحى ROC.
\end{abstract}

\begin{abstract}
ABSTEACT:
The research aims to predict financial crises in the Iraqi banks using neural networks, where the application has been using a sample of Iraqi banks during the period (2007- 2015) using a set of financial ratios (assets to equity, return on assets before tax, net profit to total assets. ..).

The research has come to the importance of using neural networks to predict the financial crisis, reaching the area under the Receiver Operator Characteristic (ROC) curve (0.975) indicating a high accuracy.
\end{abstract}

Key Words: neural networks, financial crises, predict, ROC curve.

مقدمة:

تجنب الأزمات وتخفيض شدة الأثر فى حالة حدوثها، ويمنل ذلك أهمية

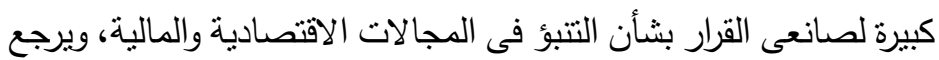

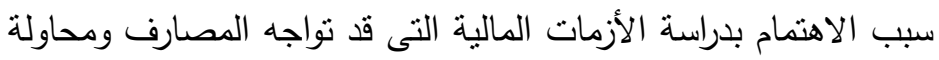

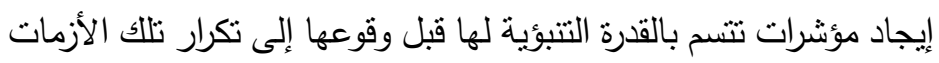
وفى فترات منقاربة من ناحية، ومن ناحية أخرى إلى الخسائر والتكلفة العالية لتلك الأزمات المتمنلة فى الخسائر من الناتج المحلى الإجمالى.

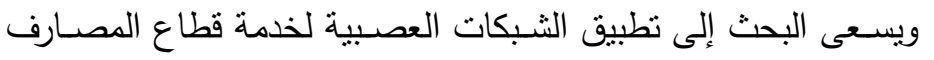

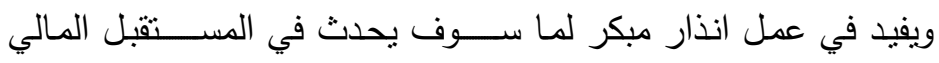
للمصــــارف حتى يمكن تجنب المشـــــلات من بدايتها وليس انتظـاراً

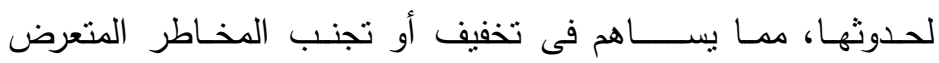
لها وتفادي حدوث منل هذه الأزمات في المستقبل
تحتل الصناعة المصرفية أهمية كبيرة في الحياة الإقتصادية

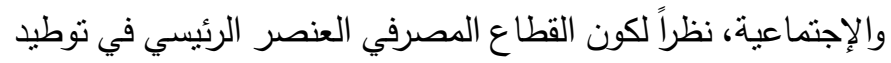

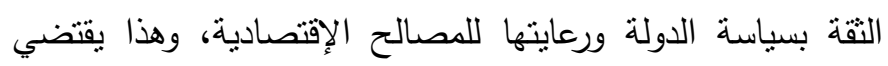
العمل على إيجاد قطاع مصرفي قوي يساعد على إمداد القطاعات

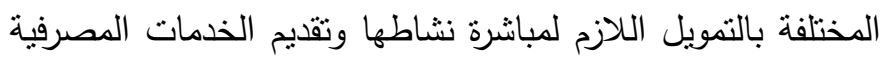

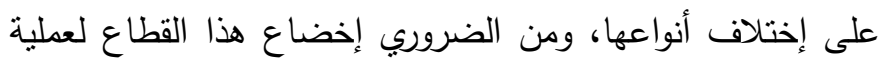

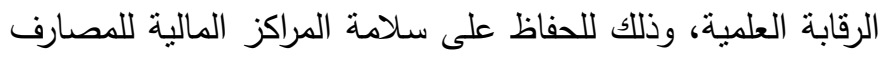
والتوصل إلى قطاع مصرفي سليم، يحافظ على حقوق المودعين

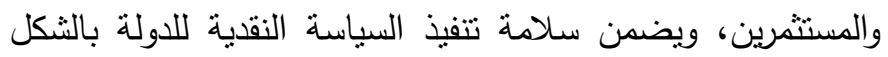
المناسب، للمساهمة بشكل فعّال في تطور الإقتصاد الوطني وإزدهاره.

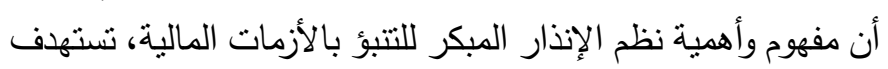


يهدف البحث إلى مقارنة بين اسلوبى التحليل التميزي والثبكات العصبية

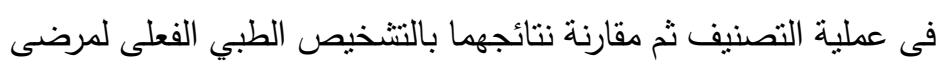

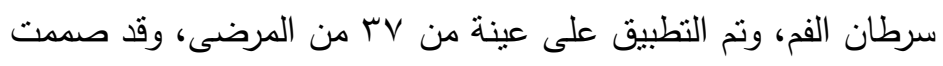

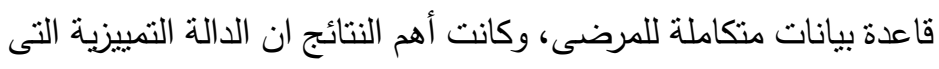

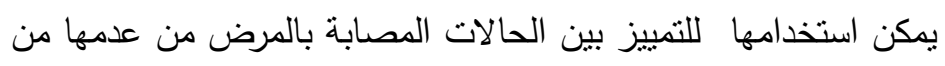

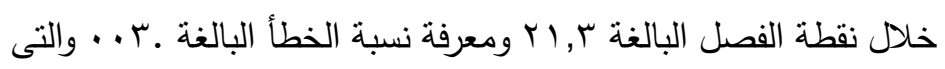

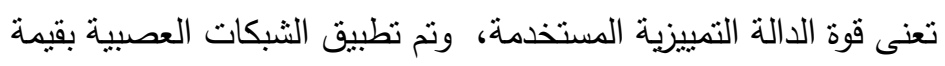

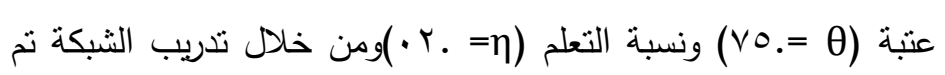

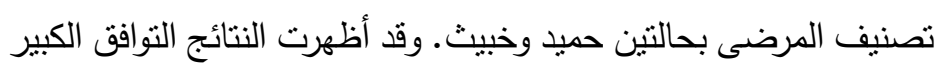
بين نتائج التصنيف باستخدام التحليل التمبيزى والثبكات العصبية مع وخين التشخيص الطبى.

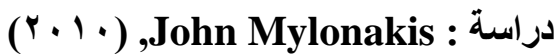
تتاولت هذه الدراسة احتمالية استخدام تحليل التمايز الخطي كنموذج لتقييم الائتمان المصرفي في المصارف التجارية، وحاولت هذه الدراسة الإجابة

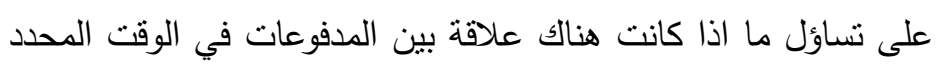

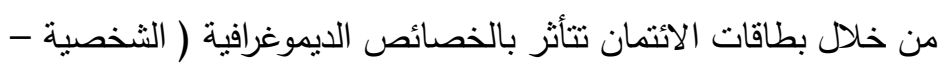
الوضع العائلي). استخدمت الدراسة تحليل التمايز بين عملاء بطاقات الائتمان ودراسة ما

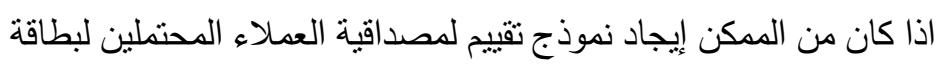

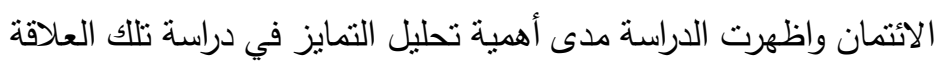

\section{دراسة: Holden and Kelley} تتاولت هذه الدراسة تأثير نوعين من أخطاء سوء التصنيف، سوء

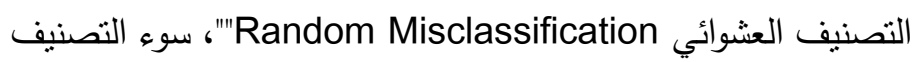
غير العشوائي "Nonrandom Misclassification" على الاداء التصنيفي لدالة تحليل التمايز بنوعيها الخطي والتربيعي وكذلك النماذج

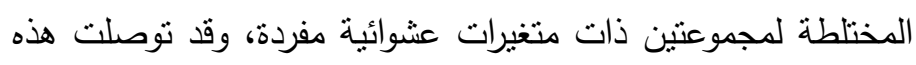

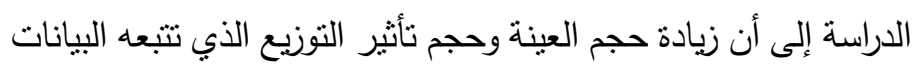
يؤدي الى زيادة دقة التصنيف.

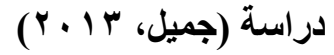
التوصل لدالة التمايز لعناصر اللياقة البدنية للاعبى كرة البد، واستملت

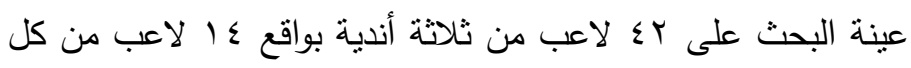
فريق بعد استبعاد حارسى المرمى، وباستخدام الاستبيان والاختبارات

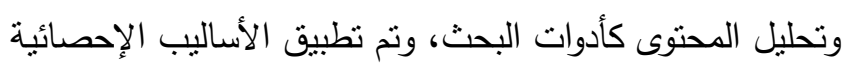

يلعب القطاع المصرفي دورا هاما في الحياة الاقتصادية والاجتماعية، نظرا لكونه العنصر الرئيسي في توطيد الثقة بسياسة الدولة ورعايتها

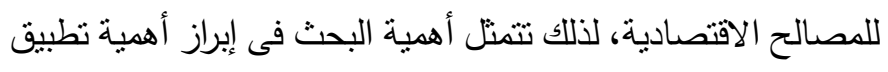
Artificial Neural عم الاحصاء منتل فى الثبكات العصبية ANN Network وتوضيح قدرته على حل المشكلات المتعلقة بهذا المجال.

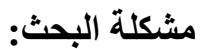
تتمنل مشكلة البحث في محاولة الباحث استخدام أسلوب علمي

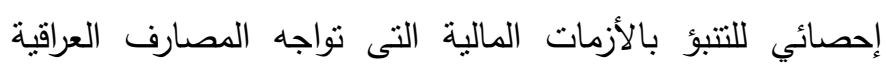
والإنذار المبكر لتلك الأزمات.

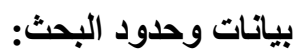
يرتكز مجال البحث على عينة عشوائية من المصارف العراقية النى اليحث تمنل المصارف الملتزمة بتطبيق مقررات لجنة بازل حيث يتم الاستفادة

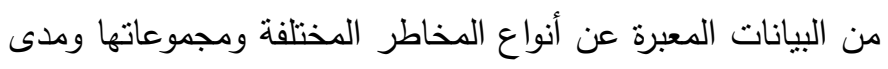

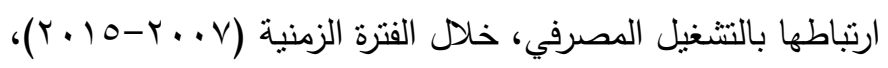
من خلال مجموعة من النسب المالية (الأصول إلى حقوق الملكية،

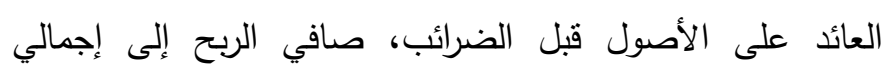

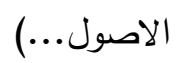
الار اسات السابقة: دراسة (محمد, 2005) هدفت هذه الدراسة الى استخدام أنموذج لوغاريتم النسبة المضافة

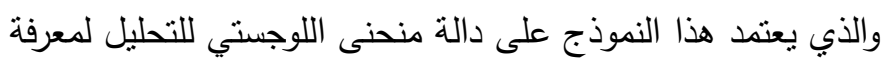
مدى نجاح او فنثل الثركات ,وقسمت الدراسة الشركات الصناعية الى ثناث اقسام الثركات صناعة المواد الانشائية وشركات الصناعة الثنات

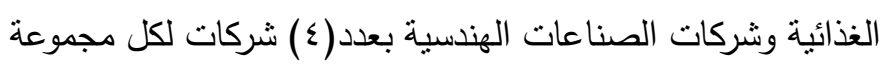
والتي تهدف الى معرفة مدى تأثز مستويات ادائها بالمتغيرات المالية

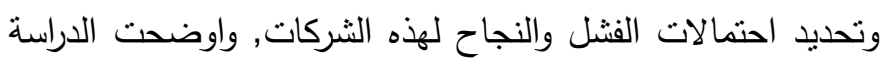
ان منوسط احتمال النجاح كانت عالية في مجموعة شركات

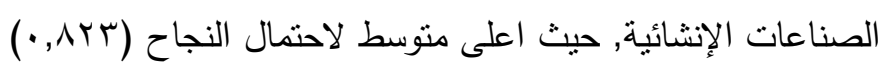

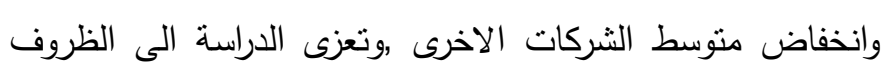
الاقتصادية العامة هي السبب الأول في انحسار نشاط هذه الثركات,

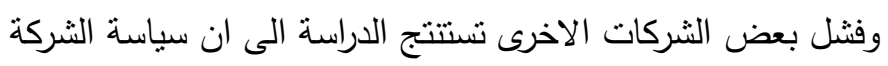

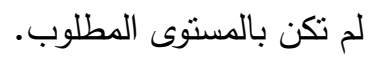




\begin{tabular}{|c|c|}
\hline 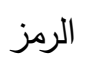 & 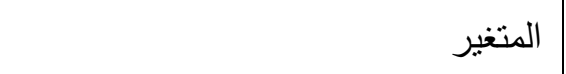 \\
\hline$x$ & الأصول الى حقوق الملكية \\
\hline$r X$ & حقوق الملكية الى الالتزامات قصيرة الأجل \\
\hline$r X$ & هامش مجمل الربح \\
\hline$\leqslant X$ & معدل دوران الأصول الثابتة \\
\hline$\circ x$ & صافي الربح الى اجمالي الاصول \\
\hline 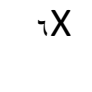 & معدل العائد على الودائع \\
\hline $\mathrm{x}$ & معدل العائد على القروض \\
\hline
\end{tabular}

ا ـ هيئة الأوراق المالية العراقية (www.isc.gov.iq). r. التقارير والقوائم المالية المنشورة من قبل المصارف العراقية. خطة البحث. يمكن إيجاز خطة البحث في البهن العناصر التالية كما يلى: أولا: الثبكات العصبية. ثانياً: التببؤ باستخدام الثبكات الثبية العصبية ثالثأ: عرض لأهم التتائج والتوصيات.

أولا: الثبكات العصبية:

الثبكات العصبية Artificial Neural Network ANN، هى محاولة وضـع نموذج يحاكي النظام العصبي البيولوجي الموجود في دماغ الإنسان لغرض معالجة المعلومات من خلال بناء نظام هيكلي جديد يقوم بربط العديد من عناصر المعالجة ونتظيمها وهي العصبونات (Neurons) المرتبطة مع بعضها وتعمل بشكل منتاسق لحل الظاهرة محل الدراسة، الظفيري ، (ع · . ץ) ، بيداء(2001) ، غولى، الحمدانى (2010)، ZZoua (2007). ،Evans, (2013) Tkacz (2001) لنموذج الثبكات العصبية ANN الذى يأخذ الشكل: $\left.y_{t}=F[H) \wedge y_{t-1},(H) r y_{t-2}, \ldots,\left(H_{N}\right) y_{t-n}\right]+U$

Dependent variable تمنل المتغير التابع : y

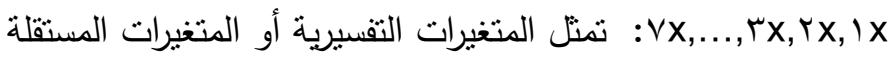
Independent variables H ،F تمثل دوال الثبكات العصبية :F

Urror Term نمثل حد الخطأ في الدالة وحسب لغة الشبكات العصبية يكون : Input بالمدخلات i x تسمى مجموعة تuمى المتغير y بالمخرجات Output
تهدف الدراسة إلى ( الوسط الحسابى والانحراف المعياري ومعامل الارتباط وتحليل الانحدار وتحليل التمايز)، وتم التوصل الى ان الاختبار يميز بين اللاعبين وأوصى البحث باستخدام الدالة التميزية فى التبؤ والتصنيف بين لاعبى اليد. دراسة تتاولت هذه الدراسة شرح الملامح الرئيسية لمستويات ربحية المصارف التي تتحصر في منغيرين هما الربح والخسارة بناء على دراسات معدل العائد على حقوق المساهمين، وركزت الدراسة على الاختبار الاحصائي باستخدام تحليل التمايز من خلال مجموعات منتوعة من المؤثرات المالية تحدد مدى ربحية المصارف ولها علاقة بها. وتوصلت النتائج الى أن التحليل المميز طريقة إحصائية ناجحة ومناسبة لحل مشكلات التقدم الى الامام دون مخاوف الإفلاس نظراً لأنه بساعد في التتبؤ باتجاهات العائد على الاستثمار التي تحلل اتجاهات الربحية. مما سبق يتضح استخدام تحليل التمايز فى المجالات المتعددة ومنها الطبي والرياضى وكذلك العلوم الإدارية كالمحاسبة والإدارة فى حالة تصنيف الثركات من رابحة وخاسرة وتحسين غش المراجعة، وفى هذا البحث يتم تطبيق الثبكات العصبية فى التبؤ بالأزمات المالية التى تواجه المصارف العراقية والإنذار المبكر لتلك الأزمات. المتغير ات والرموز تم استخدام المؤشرات المالية الخاصة بالمخاطر المصرفية حيث تم إستخدام V متغيرات مستقلة خاصة بالمخاطر وهي كالآتي: والمتغير التابع: y يأخذ القيمة ( • ) فى حالة عدم وجود أزمات مالية، والقيمة ( (1) فى حالة وجود أزمات مالية) المصارف محل الاراسة: مصارف لايوجد فيها مخاطر مالية: مصرف بغداد • مصرف الخليج التجاري • مصرف الثرق الأوسط العراقى للإسنثمار مصرف الثمال للتمويل والاستثمار

مصارف يوجد فيها مخاطر مالية: مصرف الاقتصاد للاستثمار والتمويل ماله:

$$
\begin{aligned}
& \text { •مصرف سومر التجاري } \\
& \text { مصرف بابل } \\
& \text { مصرف الأهلي العراقي }
\end{aligned}
$$


تسمى Hidden Layer بدوال التحفيز للطبقات الخفية Hiات المرحلة الثانية: تدريب الشبكة:

ينت تدريب الثبكة عدد من المرات حتى ينت الحصول على أقل خطأ Activation Function ممكن، وقد تم استخدام التدريب الإشرافى Supervised Training حيث يتم التدريب عن طريق نقديم تتابع من منجهات التدريب كمدخلات

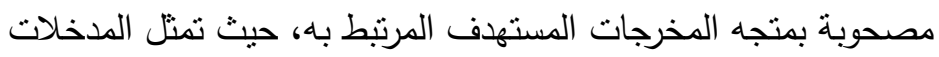

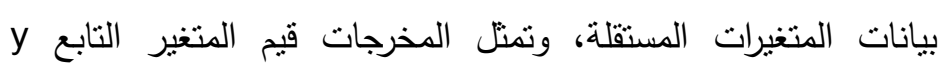

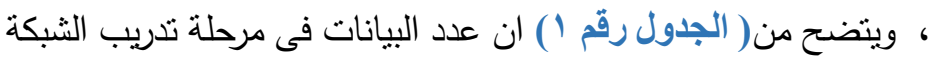

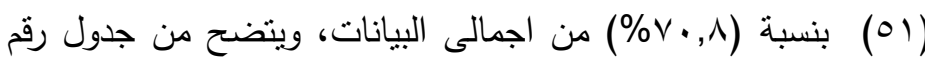

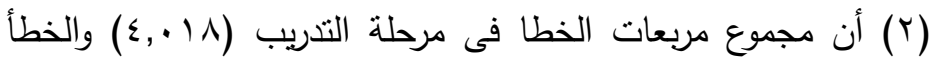

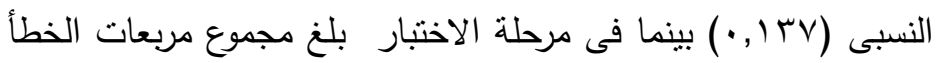

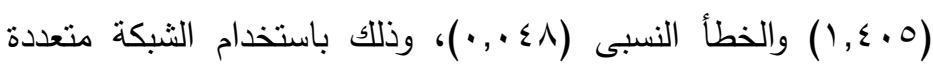
(Multilayer Perceptron (MLP . الطبقات

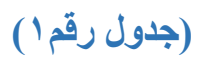
ملخص الحالات في التّريب والاختبّار

\begin{tabular}{|c|c|c|}
\hline النسبة \% & العدد & \\
\hline$\vee \cdot, \Lambda$ & 01 & التدريب \\
\hline$r q, r$ & YI & الاختبار \\
\hline \multirow[t]{3}{*}{$1 \cdots}$, & Vr & المجموع \\
\hline & $\cdot$ & المستبعد \\
\hline & $V Y$ & الاجمالى \\
\hline
\end{tabular}

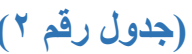

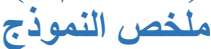

\begin{tabular}{|c|c|c|}
\hline الخطأ النسبى & مجموع مربعات الخطأ & \\
\hline$\cdot, I T V$ & $\varepsilon, \cdot 11$ & التدريب \\
\hline$\cdot, \cdot \leq \wedge$ & $1, \varepsilon .0$ & الاختبار \\
\hline
\end{tabular}

يعرض (جدول رقم ب) و (شكل رقم ץ) الأهمية النسبية للمتغيرات المستقلة فى نموذج الثبكات العصبية ويتضح من الجدول ارتفاع الأهمية

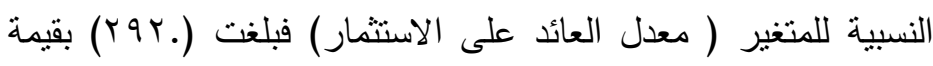

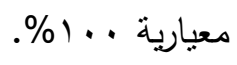

$y=1 /\left(1+e^{-t}\right)$

وتتمثل مراحل استخدام الشبكات العصبية فيمايلى: المرحلة الأولى : تحديد هيكل الثبكة: تم استخدام برنامج SPSS ver. فى تحديد هيكل الثبكة

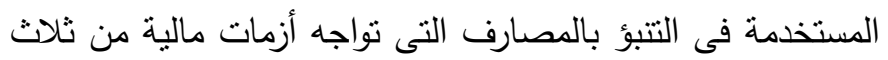
طبقات، الطبقة الأولى وهى طبقة المدخلات وتتمثل فى النسب المالية للبنوك محل الدراسة خلال فترة الدراسة، والطبقة الثانية هى الطبقة الخفية وتتكون من نيورون واحد، بينما الطبقة الثالثة هى طبقة

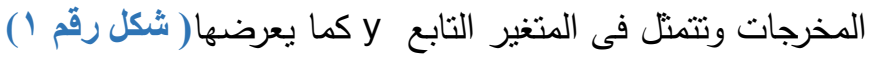

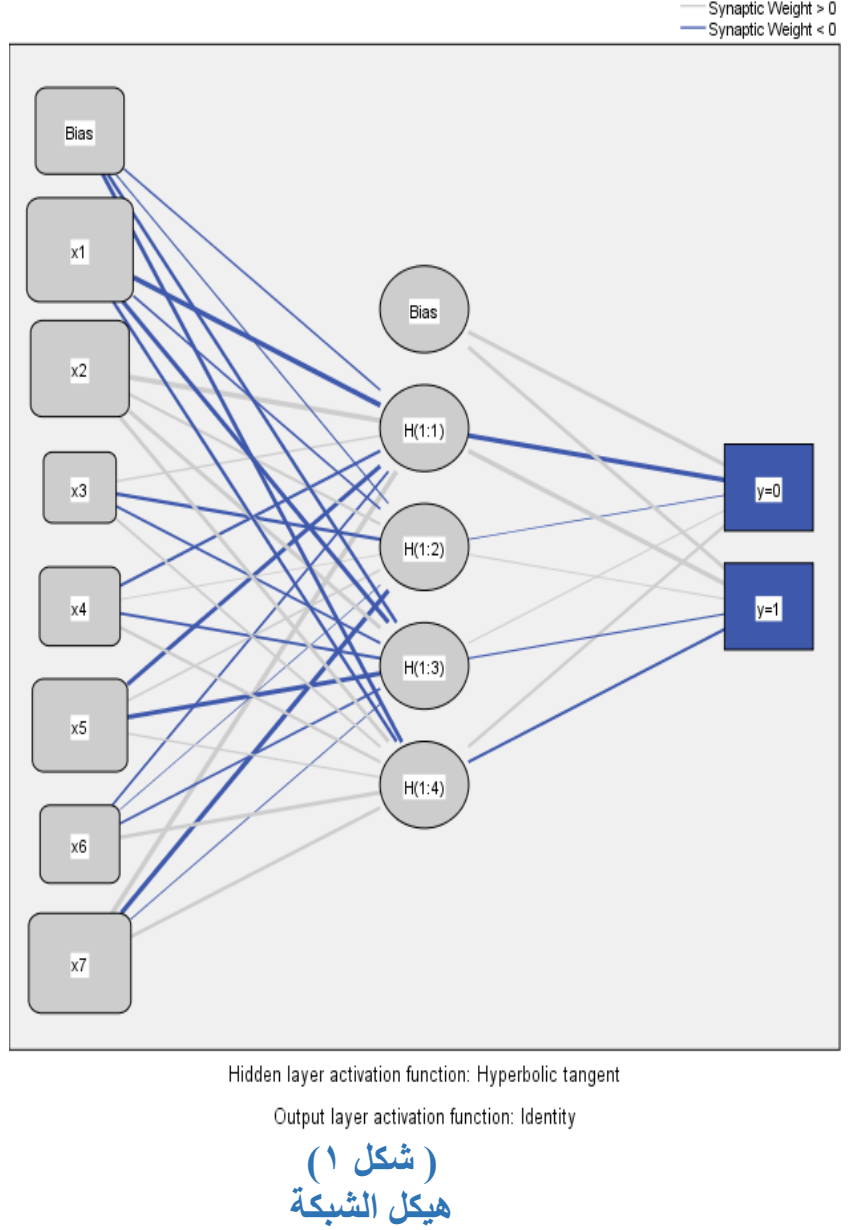


(جدول رقّم ع (2018)

تقديرات MLP لنموذج الثبكات العصبية

\begin{tabular}{|c|c|c|c|c|c|c|c|}
\hline \multicolumn{6}{|c|}{ Predicted } & \multirow{3}{*}{\multicolumn{2}{|c|}{ Predictor }} \\
\hline \multicolumn{2}{|c|}{ Output Layer } & \multicolumn{4}{|c|}{ Hidden Layer 1} & & \\
\hline$[y=1]$ & {$[y=0]$} & $H(1: 4)$ & $H(1: 3)$ & $H(1: 2)$ & $\mathrm{H}(1: 1)$ & & \\
\hline & & $\varepsilon 0 \varepsilon$. & ArE. & $00 V_{\text {. }}$ & . $\varepsilon \varepsilon .-$ & Bias) & \multirow{8}{*}{ nput|Layer } \\
\hline & & $\lambda \varepsilon r_{.-}$ & rok. & $r, 00 \varepsilon$ & $\varepsilon \forall V_{.}$ & $\mathrm{x} 1$ & \\
\hline & & $1,+19$ & $-1 .-$ & $1,+\times 1$ & 177 & $\mathrm{x} 2$ & \\
\hline & & THT. & rT9.- & 1.r. & $15 T .-$ & x3 & \\
\hline & & $r A Y_{-}$ & rio. & 117. & or. & $x 4$ & \\
\hline & & .11 & $\varepsilon \cdot v$. & $\varepsilon \vee \varepsilon$ & $91 \varepsilon$. & $\mathrm{x5}$ & \\
\hline & & $\varepsilon \pi I_{-}$ & $\cdot \vee \vee \varepsilon$. & ro9. & TrE. & $x 6$ & \\
\hline & & $1,1 \times V$ & $.1 \%$. & T,9Tr. & Tro.- & $x 7$ & \\
\hline$\varepsilon \pi$ & $\varepsilon \wedge T$ & & & & & (Bias) & \multirow{5}{*}{ Hidden Layer 1} \\
\hline 017 & $\varepsilon r$. & & & & & $H(1: 1)$ & \\
\hline $1, .19$ & $9 r \times$ & & & & & $H(1: 2)$ & \\
\hline .0 & .1. & & & & & $H(1: 3)$ & \\
\hline 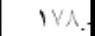 & 150 & & & & & $H(1: 4)$ & \\
\hline
\end{tabular}

عرض (جدول رقم \&) نقديرات MLP لنموذج الثبكات العصبية، وتظهر النتائج قيمة الأوزان من المدخلات إلى الطبقة المخفية ومن

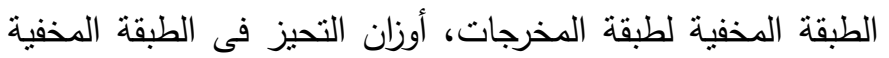

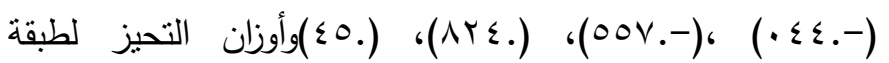

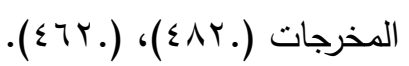
المرحلة الثالثة: التنبؤ: تم استخدام بيانات التدريب فى عملية التتبؤ، وقد تم التتبؤ بتصنيف المصارف من حيث التعرض لأزمات مالية، يوضح (جدول رقمه ) تصنيف المصارف:

\begin{tabular}{|c|c|c|c|}
\hline المصرف & السنة & الألتابع التعمات & $\begin{array}{l}\text { MLP_ } \\
\text { Predicted } \\
\text { Value }\end{array}$ \\
\hline بغداد & 2013 & 0 & 0 \\
\hline بغداد & 2014 & 0 & 0 \\
\hline بغداد & 2015 & 0 & 0 \\
\hline الخليج التجاري & 2007 & 0 & 0 \\
\hline الخليج التجاري & 2008 & 0 & 0 \\
\hline الخليج التجاري & 2009 & 0 & 0 \\
\hline الخليج التجاري & 2010 & 0 & 0 \\
\hline الخليج التجاري & 2011 & 0 & 0 \\
\hline الخليج التجاري & 2012 & 0 & 0 \\
\hline
\end{tabular}

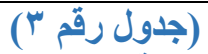

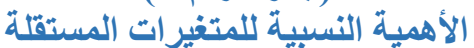

\begin{tabular}{|c|c|c|}
\hline $\begin{array}{l}\text { Normalized } \\
\text { Importance }\end{array}$ & $\begin{array}{c}\text { Importance } \\
\text { MLP }\end{array}$ & \\
\hline $1 \cdots$, & rq৭. & الأصول الى حقوق الملكية \\
\hline $0 \leqslant, r$ & 174. & الالتزامات قصيرة الملكية الى الأجل \\
\hline$r, v$ & .11. & هامش مجمل الربح \\
\hline r), r & . & معدل دوران الأصول الثابتة \\
\hline $0 \leqslant, 1$ & $17 r$. & 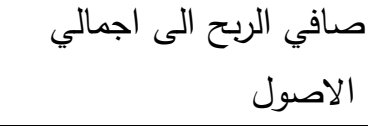 \\
\hline IT,V & . $\leqslant$. & معدل العائد على الودائع \\
\hline$\wedge \vee, \vee$ & rTr. & معدل العائد على القروض \\
\hline
\end{tabular}

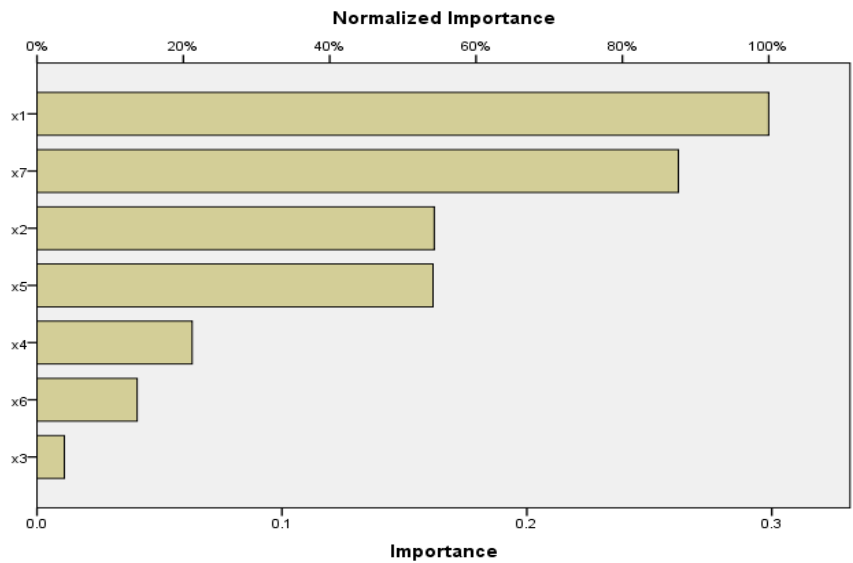

(شُكل رقم ץ')

الأهمية النسبة للمتغيرات المستقلة

\begin{tabular}{|c|c|c|c|}
\hline المصرف & السنة & الأزمات التعترض & $\begin{array}{c}\mathrm{MLP}_{-} \\
\text {Predicted } \\
\text { Value }\end{array}$ \\
\hline بغداد & 2007 & 0 & 0 \\
\hline بغداد & 2008 & 0 & 0 \\
\hline بغداد & 2009 & 0 & 0 \\
\hline بغداد & 2010 & 0 & 0 \\
\hline بغداد & 2011 & 0 & 0 \\
\hline بغداد & 2012 & 0 & 0 \\
\hline
\end{tabular}




\begin{tabular}{|c|c|c|c|}
\hline المصرف & السنة & الألتابع التعمات & $\begin{array}{l}\mathrm{MLP}_{-} \\
\text {Predicted } \\
\text { Value }\end{array}$ \\
\hline سومر التجاري & 2009 & 1 & 1 \\
\hline سومر التجاري & 2010 & 1 & 1 \\
\hline سومر التجاري & 2011 & 1 & 1 \\
\hline سومر التجاري & 2012 & 1 & 1 \\
\hline سومر التجاري & 2013 & 1 & 1 \\
\hline سومر التجاري & 2014 & 1 & 1 \\
\hline سومر التجاري & 2015 & 1 & 1 \\
\hline بابل & 2007 & 1 & 1 \\
\hline بابل & 2008 & 1 & 1 \\
\hline بابل & 2009 & 1 & 1 \\
\hline بابل & 2010 & 1 & 1 \\
\hline بابل & 2011 & 1 & 1 \\
\hline بابل & 2012 & 1 & 1 \\
\hline بابل & 2013 & 1 & 1 \\
\hline بابل & 2014 & 1 & 1 \\
\hline بابل & 2015 & 1 & 1 \\
\hline الأهلي العراقي & 2007 & 1 & 1 \\
\hline الأهلي العراقي & 2008 & 1 & 1 \\
\hline الأهلي العراقي & 2009 & 1 & 1 \\
\hline الأهلي العراقي & 2010 & 1 & 1 \\
\hline الأهلي العراقي & 2011 & 1 & 1 \\
\hline الأهلي العراقي & 2012 & 1 & 1 \\
\hline الأهلي العراقي & 2013 & 1 & 1 \\
\hline الأهلي العراقي & 2014 & 1 & 1 \\
\hline الأهلي العراقي & 2015 & 1 & 0 \\
\hline
\end{tabular}

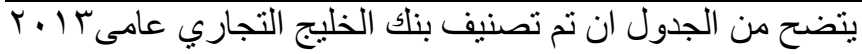

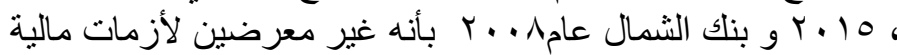

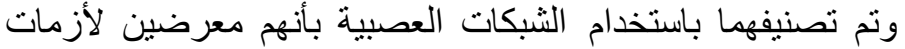

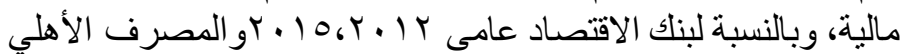

\begin{tabular}{|c|c|c|c|}
\hline المصرف & السنة & (التألتعرض & $\begin{array}{c}\mathrm{MLP}_{-} \\
\text {Predicted } \\
\text { Value }\end{array}$ \\
\hline الخليج التجاري & 2013 & 0 & 1 \\
\hline الخليج التجاري & 2014 & 0 & 0 \\
\hline الخليج التجاري & 2015 & 0 & 1 \\
\hline الثرق الأوسط & 2007 & 0 & 0 \\
\hline الثرق الأوسط & 2008 & 0 & 0 \\
\hline الثرق الأوسط & 2009 & 0 & 0 \\
\hline الشرق الأوسط & 2010 & 0 & 0 \\
\hline الثرق الأوسط & 2011 & 0 & 0 \\
\hline الثرق الأوسط & 2012 & 0 & 0 \\
\hline الثمال & 2007 & 0 & 0 \\
\hline الثمال & 2008 & 0 & 1 \\
\hline الشمال & 2009 & 0 & 0 \\
\hline الثمال & 2010 & 0 & 0 \\
\hline الشمال & 2011 & 0 & 0 \\
\hline الثمال & 2012 & 0 & 0 \\
\hline الشمال & 2013 & 0 & 0 \\
\hline الثمال & 2014 & 0 & 0 \\
\hline الثمال & 2015 & 0 & 0 \\
\hline الأقتصاد & 2007 & 1 & 1 \\
\hline الأقتصاد & 2008 & 1 & 1 \\
\hline الأقتصاد & 2009 & 1 & 1 \\
\hline الأقتصاد & 2010 & 1 & 1 \\
\hline الأقتصاد & 2011 & 1 & 1 \\
\hline الأقتصاد & 2012 & 1 & 0 \\
\hline الأقتصاد & 2013 & 1 & 1 \\
\hline الأقتصاد & 2014 & 1 & 0 \\
\hline الأقتصاد & 2015 & 1 & 1 \\
\hline سومر التجاري & 2007 & 1 & 1 \\
\hline سومر التجاري & 2008 & 1 & 1 \\
\hline
\end{tabular}




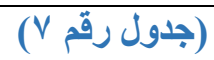

التنبؤ باستخدام الشبكات العصية

\begin{tabular}{|l|l|r|l|l|l|l|r|}
\hline$X_{1}$ & $X_{2}$ & \multicolumn{1}{|l|}{$X_{3}$} & $X_{4}$ & $X_{5}$ & $X_{6}$ & $X_{7}$ & (i) \\
\hline 3.7475 & 0.3815 & 0.6206 & 0.5982 & 0.0385 & 0.0472 & 0.3866 & 0 \\
\hline 1.7132 & 1.3601 & 0.821 & 3.2255 & 0.0135 & 0.0268 & 0.2199 & 1 \\
\hline 1.5751 & 1.7232 & 0.4078 & 0.9324 & 0.0041 & 0.0957 & 0.1268 & 1 \\
\hline 3.5413 & 0.3942 & 0.7384 & 0.7044 & 0.0389 & 0.0485 & 0.4074 & 0 \\
\hline
\end{tabular}

ثالثاً: النتائج والتوصيات:

! توصل البحث إلى أهمية الثبكات العصبية العبية متعددة

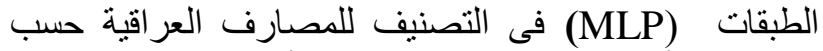

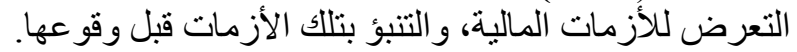

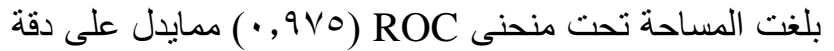
عالية للشبكات العصبية فى التصنيف.

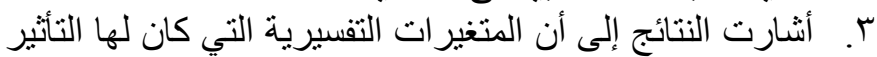

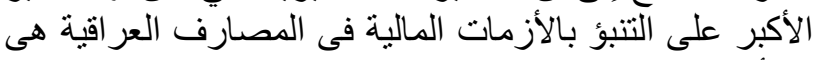

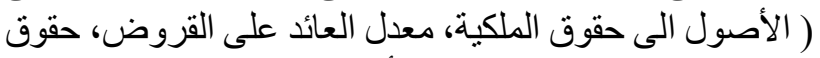
الملكية الى الالتز امات قصيرة الألى الأل، صافي الربح إلى إجمالي

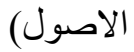
؛. يوصى البحث يوصى باستخدام نماذج الثبكات العصبية

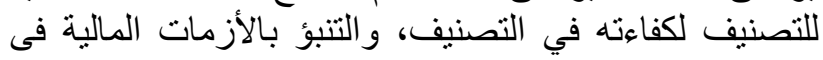

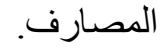
كما يوصى البحث بإمكانية التطبيق على كافة المصارف العبار العر اقية وزيادة النسب المالية المستخدمة فى التحليل.

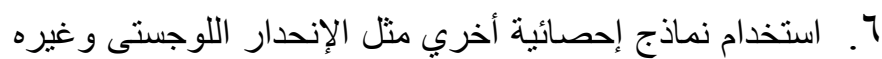
من الأساليب الإحصائية وتطبيقه على إحصى بيانات البحث ومقار الإنتها

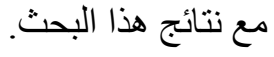

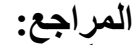

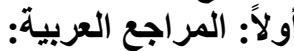

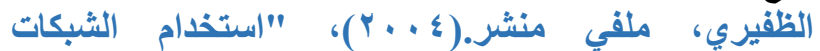

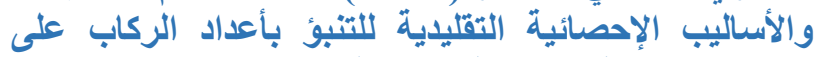

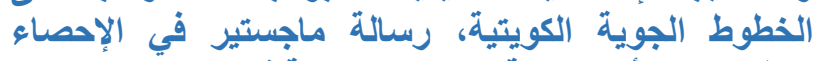

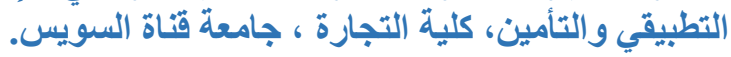

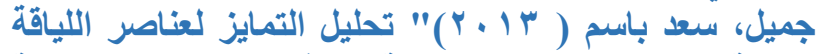

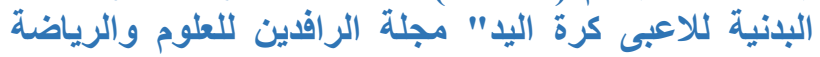

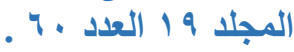

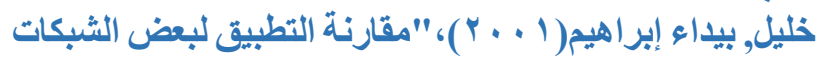

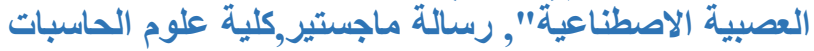

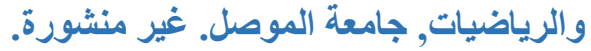

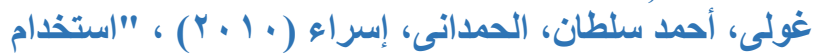

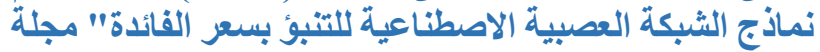

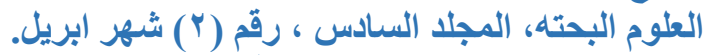

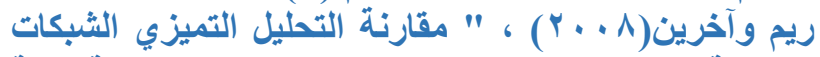

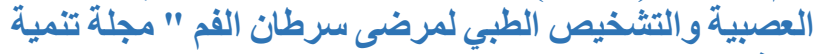

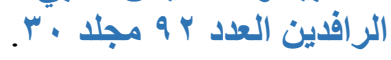

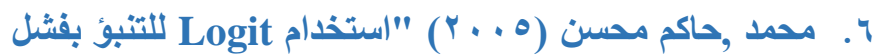

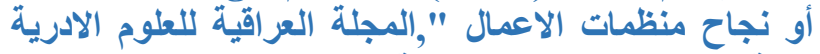

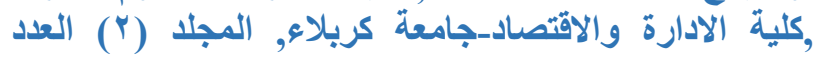

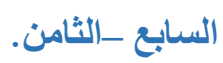

$\cdot r$ .0

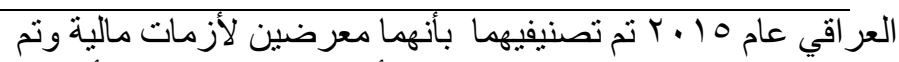
تصنيفيهما باستخدام الثبكات العصبية بأنهما غير معرضين لأنين لأزمات

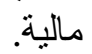

: ROC منحنى

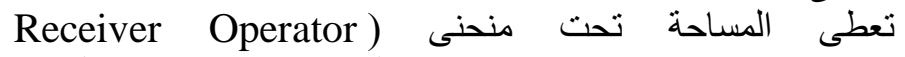
Characteristic Curve) ROC

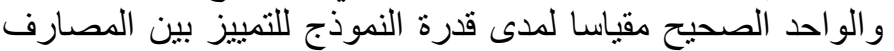

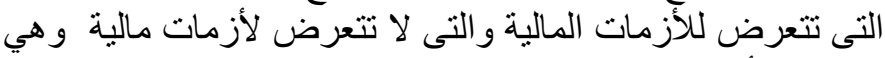

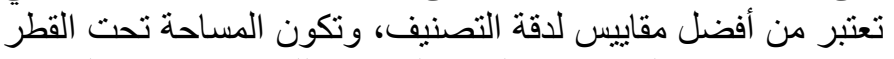

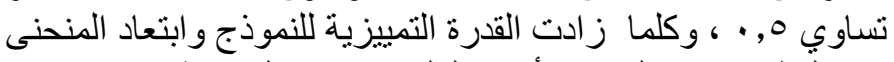

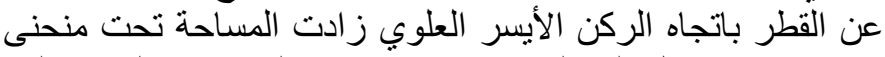
ROC حتى تصل الى القيمة واحد صحيح والتي تعني التمييز التام التئي

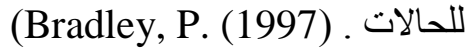

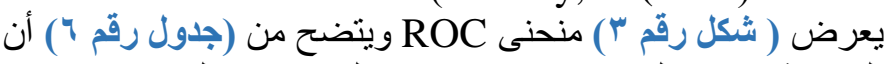

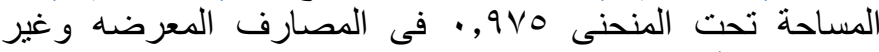

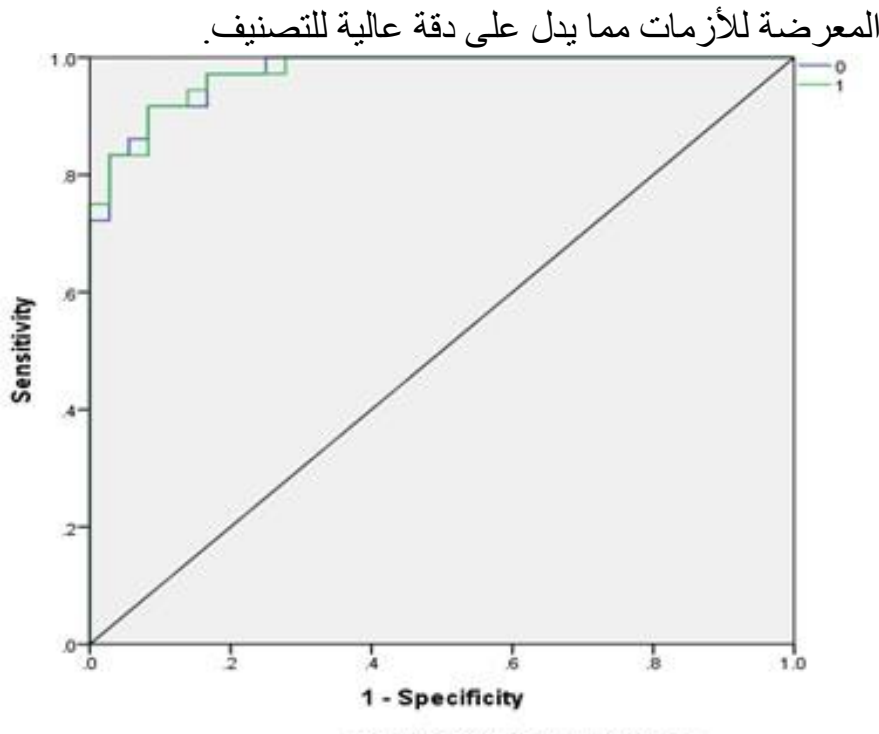

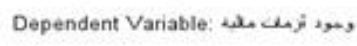

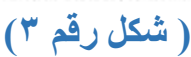

منحنى ROC لحسنسية النموذج

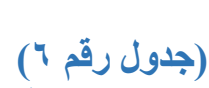

\begin{tabular}{|c|c|c|}
\hline & & المساحة \\
\hline \multirow{2}{*}{ وجود أزمات مالية } & لا يوجد & .975 \\
\hline & يوجد & .975 \\
\hline
\end{tabular}

ثانياً: التنبؤ باستخدام الثبكات العصبية:

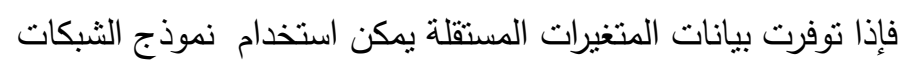

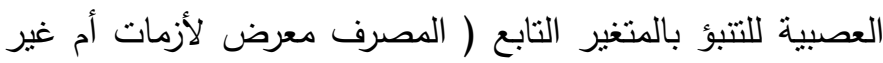

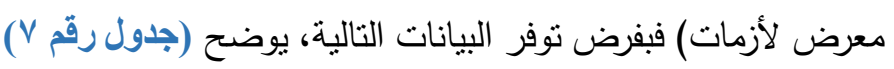

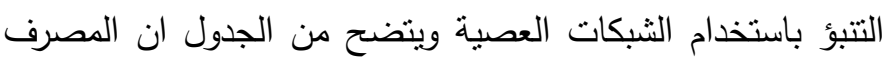

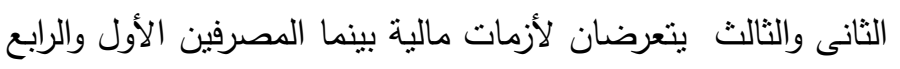
لا يتعرضان لأزمات مالية.

Delta University for Science and Technology

Coastal International Road, Mansoura, Gamasa City, Dakahlia, Egypt

E-mail: dusj@deltauniv.edu.eg

Journal homepage: www.deltauniv.edu.eg/dusj 


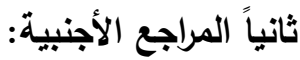

1. Abhishek S., Mishra G. C (2015) " Application of Box-Jenkins method and Artificial Neural Network procedure for Time Series Forecasting of Prices" Statistics in Transition, new series, Spring ,Vol. 16, No. 1, pp. 83-96 .

2. Bradley, P. (1997). "The Use of the Area under the ROC Curve in the Evaluation of Machine Learning Algorithms. Pattern Recognition. Vol.30, No.7, pp.1145-1159.

3. Evans, c., (2013) " Utilizing artificial neural networks and genetic algorithms to build an algotrading model for intra-day foreign exchange speculation" Faculty of Technology, Engineering and the Environment School of Computing, Telecommunications and Networks Birmingham City University, UK.

4. Holden, Jocelyn, Kelley, Ken ,(2010), " The Effects of Initially Misclassified Data on the Effectiveness of Discriminant Function Analysis and Finite Mixture Modeling", Educational and Psychological Measurement, Vol. 70,pp.36-55.

5. John Mylonakis, (2010)," Evaluating the Likelihood of Using Linear Discriminant Analysis as A Commercial Bank Card Owners Credit Scoring Model" International Business Research Vol. 3, No.2,pp.9-20.

6. Mohamed M.M. (2010), " Forecasting stock exchange movements using neural networks: empirical evidence from Kuwait, Expert Systems with Applications" , vol. 27, no. 9, pp.6302-6309.

7. Rozga Ante \& Kundid Ana ,(2013)," Discriminant Analysis of Bank Profitability Levels " Croatian Operational Research Review (CRORR), Vol. 4.,pp.153-163.

8. Stokes, A. (2011), "Forecasting exchange rates using neural networks: a traders approach". Student Theses \& Publication.

9. Tkacz, G., (2001), "Neural Network Forecasting of Canadian GDP Growth", International Journal of Forecasting 17, pp. ,57-69.

10. Zoua, H. F., at, el. (2007), "An investigation and comparison of artificial neural network and time series models for Chinese food grain price forecasting. Neurocomputing, 70, 2913-2923. 